\title{
A systematic approach to improve the outcomes of type A aortic dissection
}

\author{
Hidefumi Nishida, MD, ${ }^{\mathrm{a}}$ Minoru Tabata, MD, MPH, ${ }^{\mathrm{a}, \mathrm{b}}$ Toshihiro Fukui, MD, ${ }^{\mathrm{a}, \mathrm{c}}$ Yasunori Sato, PhD, ${ }^{\mathrm{d}}$ \\ Hajime Kin, MD, ${ }^{a}$ and Shuichiro Takanashi, $\mathrm{MD}^{\mathrm{a}}$
}

\section{ABSTRACT}

Objectives: The aims of this study are to evaluate the outcomes and trends of contemporary emergency surgery for acute type A aortic dissection on the basis of a systematic approach and to assess the impact of temporary aortic crossclamping during systemic cooling on early and late outcomes.

Methods: We retrospectively reviewed 702 consecutive patients who underwent emergency surgery for acute type A aortic dissection between March 2004 and May 2015. Our clinical protocol includes rapid transfer to the operating room, quick establishment of cardiopulmonary bypass, temporary aortic crossclamping during cooling, primary entry resection, and open distal anastomosis. We analyzed the perioperative data, survival, freedom from aortic reinterventions, and impact of aortic crossclamping on early and late outcomes.

Results: The median time from hospital arrival to cardiopulmonary bypass establishment was 115 minutes and has decreased over the last decade (trend test $P<.001)$. We perfused the femoral artery in 615 patients $(87.6 \%)$, placed aortic crossclamping in 616 patients $(87.7 \%)$, and performed open distal anastomosis in all patients. The operative mortality was $5.4 \%$ (38/702), and the incidence of stroke was $10.8 \%$ (76/702). The 7-year overall survival and freedom from aortic reinterventions were $80.4 \%$ and $87.5 \%$, respectively. Compared with the nonclamping group, the crossclamping group had a shorter operation time, similar operative mortality, incidence of stroke, and freedom from aortic reinterventions.

Conclusions: Emergency surgery for acute type A aortic dissection based on our systematic approach demonstrated excellent early and late outcomes. The temporary aortic crossclamping during cooling decreased the operation time without increasing early and late adverse events. ( $\mathrm{J}$ Thorac Cardiovasc Surg 2017;154:89-96)

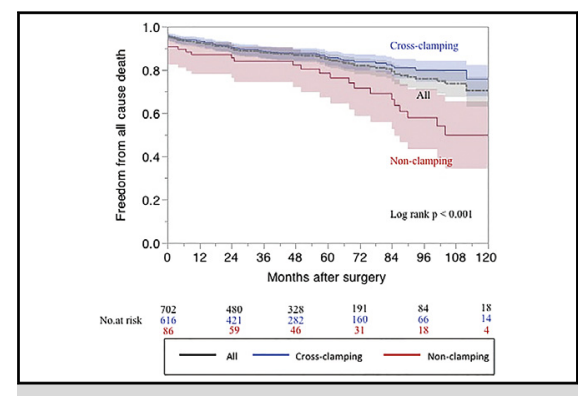

Long-term survival after emergency surgery for ATAAD.

Central Message

Emergency surgery for ATAAD based on our systematic approach demonstrated excellent early and late outcomes.

\section{Perspective}

Our findings increase surgeons' awareness of the importance of a systematic approach to emergency surgery for ATAAD, which may contribute to an improvement in surgical outcomes of ATAAD. The spread of temporary aortic crossclamping during systemic cooling may shorten the average operation time without increasing adverse events.

See Editorial Commentary page 97.
Despite recent advances in surgical techniques and perioperative management, the surgical mortality of acute type A aortic dissection (ATAAD) remains high $(12.7 \%$ to $29.8 \%$ ). ${ }^{1-5}$

To improve surgical outcomes, various techniques have been developed over time. ${ }^{6}$ The current consensus about

\footnotetext{
From the a Department of Cardiovascular Surgery, Sakakibara Heart Institute; ${ }^{\mathrm{b}}$ Department of Cardiovascular Surgery, Tokyo Bay Urayasu-Ichikawa Medical Center, Tokyo; ${ }^{\mathrm{c}}$ Department of Cardiovascular Surgery, Kumamoto University, Kumamoto; and ${ }^{\mathrm{d} D e p a r t m e n t}$ of Global Clinical Research, Graduate School of Medicine, Chiba University, Chiba, Japan.

Received for publication July 13, 2016; revisions received Jan 23, 2017; accepted for publication March 9, 2017; available ahead of print April 14, 2017.

Address for reprints: Minoru Tabata, MD, MPH, Department of Cardiovascular Surgery, Tokyo Bay Urayasu-Ichikawa Medical Center, 3-4-32 Todaijima, Urayasu-Shi, Chiba 279-0001, Japan (E-mail: mtabata@post.harvard.edu). $0022-5223 / \$ 36.00$

Copyright (C) 2017 by The American Association for Thoracic Surgery http://dx.doi.org/10.1016/j.jtcvs.2017.03.050
}

the surgical technique for ATAAD recommends open distal anastomosis with hypothermic circulatory arrest. ${ }^{7-9}$ However, it is not clearly understood whether temporary aortic crossclamping during systemic cooling affects surgical outcomes or not.

The aims of this study are to evaluate the outcomes and trends of emergency surgery for ATAAD in a Japanese high-volume aortic center and to assess the impact of temporary aortic crossclamping on early and late
Scanning this QR code will take you to supplemental table and video for this article.

\section{outcomes.}

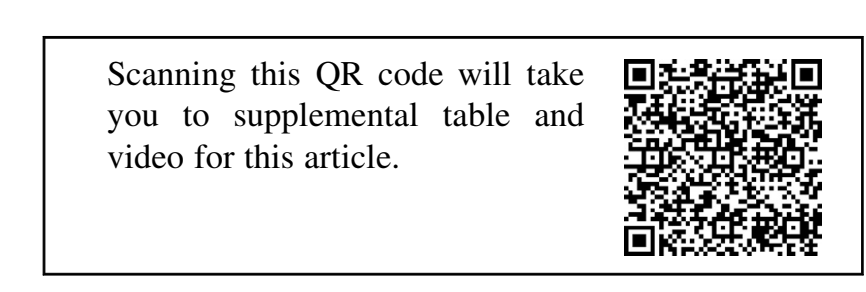




\section{Abbreviations and Acronyms}

ATAAD $=$ acute type A aortic dissection

$\mathrm{CT}=$ computed tomography

$\mathrm{CPB}=$ cardiopulmonary bypass

FA $\quad=$ femoral artery

$\mathrm{OR}=$ operating room

TEE $=$ transesophageal echocardiography

\section{MATERIALS AND METHODS \\ Patients and Study Design}

Between March 2004 and May 2015, 702 consecutive patients underwent emergency surgical aortic repair of ATAAD at Sakakibara Heart Institute. The diagnosis of ATAAD was made with computed tomography (CT) in all patients. ATAAD was defined as any nontraumatic dissection involving the ascending aorta presenting within 14 days from symptom onset.

This is a retrospective cohort study. First, we assessed the time from hospital arrival to establishment of cardiopulmonary bypass (CPB) (door-to-CPB time), operative procedural details, operative mortality, incidence of postoperative complications, long-term mortality, and aortic reinterventions. We also assessed the trend of door-to-CPB time and operative mortality over a decade. In the door-to-CPB time analysis, we excluded 1 patient who developed ATAAD during hospitalization for Stanford type B aortic dissection.

Second, we divided our 702 cases into 2 groups, the aortic crossclamping group and the nonclamping group, and compared early and late outcomes. We also evaluated the impact of aortic crossclamping on incidence of stroke and aortic reinterventions using multivariable analyses. For comparison of operation time, isolated ascending aortic or hemiarch replacement cases were exclusively included to adjust the bias of operative procedures. We included cases in which we crossclamped the aorta and aborted the clamping with any reasons in the crossclamping group.

The institutional review board of our institution approved this study and waived the requirement for informed consent. All data were collected prospectively and reviewed retrospectively from each patient's medical chart during regular visits to the outpatients' clinic or by telephone interview. The latest follow-up was conducted between June 2014 and May 2015. The mean follow-up period was $47.5 \pm 36.0$ months after surgery.

\section{Definitions}

Emergency surgery was defined as operation within 24 hours after the diagnosis. Preoperative shock was defined as an unstable preoperative condition with at least 1 of the following factors: systolic blood pressure less than $80 \mathrm{~mm} \mathrm{Hg}$, requirement of inotropes, pericardial drainage, or cardiopulmonary resuscitation. Cerebral malperfusion was defined as newly developed neurologic deficits with cervical arterial dissection on CT images. Myocardial malperfusion was defined as new electrocardiogram change indicating myocardial ischemia, elevated myocardial enzymes, and coronary arterial dissection on echocardiography or CT images. Lower-limb malperfusion was defined as newly developed lowerlimb ischemia with symptoms and corroborative CT images. Mesenteric malperfusion was defined as newly developed acute abdomen associated with a dissected superior mesenteric artery on CT images. Operative mortality was defined as any death within 30 days after surgery or before discharge. Stroke was defined as the presence of new neurologic dysfunction lasting for more than 24 hours. High-risk patients were defined as patients with at least 1 of the following factors: age 80 years or older, preoperative shock, previous cardiac surgery, and any organ malperfusion., $5,10,11$

\section{Diagnosis and Surgical Protocols}

Our systematic protocol includes rapid transfer to the operating room (OR), quick establishment of $\mathrm{CPB}$ mainly with femoral arterial cannulation, temporary aortic crossclamping during cooling, primary entry resection, and open distal anastomosis.

When the diagnosis has been made at a transferring hospital, we start setting up the OR before the patient's arrival and transfer the patient to the OR as soon as possible after arrival. Even for undiagnosed patients, we start preparing the OR except for opening the sterile instruments and CPB circuits before arrival if the reported patient's condition is likely to be ATAAD. Then, we quickly transfer the patient to the CT suite and perform contrast-enhanced scans after arrival. As soon as the diagnosis of ATAAD is made, we continue setting up the OR and stabilize the patient in the intensive care unit until the OR is ready to accept the patient.

All operations are performed through a median sternotomy. The femoral artery (FA) is primarily selected as a cannulation site unless the CT findings show malperfusion of bilateral legs or shaggy aorta. ${ }^{12}$ For quick establishment of CPB, the sternotomy and dissection of the FA are performed simultaneously. We routinely scan the true lumen of the aorta by transesophageal echocardiography (TEE) as soon as CPB is established. If the true lumen collapses, we stop $\mathrm{CPB}$ and switch the cannulation site to the left ventricular apex. We use the EZS21A cannula (Edwards Lifesciences, LCC, Irvine, Calif) for left ventricle apex perfusion. When preoperative CT shows bilateral lower limbs malperfusion or shaggy aorta, we cannulate the left ventricular apex or right axillary artery instead of the FA. In this series, the ascending aorta was cannulated in a few cases with unclear reasons. We cannulated the right atrium for systemic venous drainage and right upper pulmonary vein for left ventricle vent.

After establishment of $\mathrm{CPB}$, the patients are cooled down to $25^{\circ} \mathrm{C}$ for circulatory arrest. During systemic cooling, we usually crossclamp the aorta, open the proximal ascending aorta, administer retrograde and selective cardioplegia, and repair the aortic root. At the moment of crossclamping, we temporarily stop perfusion, decrease the pressure of aorta to zero, and clamp the aorta very slowly to minimize the pressurization of the false lumen. At the same time, an anesthesiologist temporarily compresses the bilateral carotid arteries to avoid incidental cerebral thrombosis. Needless to say, we do not clamp the aorta when we cannulate the left ventricular apex. We carefully monitor the blood pressure of the bilateral radial arteries, bilateral cerebral oxygen saturation, and TEE findings after crossclamping. If any sign of malperfusion is found, we unclamp the aorta or change the clamping site proximally. We did not crossclamp the aorta in 86 patients. The reasons why we did not crossclamp included systemic perfusion from the left ventricular apex (60 cases), severe adhesion around the ascending aorta (12 cases), large thrombosis in the ascending aortic false lumen ( 6 cases), on-pump beating coronary artery bypass grafting performed during cooling (4 cases), large entry in ascending aorta (3 cases), and distal ascending aortic rupture ( 1 case).

In supracommissural aortic repair, the dissected aortic root is repaired with BioGlue (Cryolife Inc, Kennesaw, Ga) and double strips at the level of the sinotubular junction. At the core temperature of $25^{\circ} \mathrm{C}$, systemic circulation is arrested. In ascending aortic or hemiarch replacement, the distal end of the aorta is reinforced with double felt strips and open distal anastomosis is performed with retrograde cerebral perfusion. After distal anastomosis, systemic perfusion is resumed through the branch of the prosthesis and the proximal anastomosis is performed during rewarming (Video 1). In total arch replacement, open distal anastomosis is performed with selective antegrade cerebral perfusion. We use the MERA balloon catheter 12F cannula (MERA, Inc, Tokyo, Japan). The details of our total arch replacement have been reported. ${ }^{13}$ We perform total arch replacement when the entry is located in the aortic arch or the distal aortic arch is dilated $(>45 \mathrm{~mm}) .{ }^{14}$ We perform aortic root operations when at least 1 of the following conditions exist: intimal tear in the aortic root, extensive involvement of coronary arteries, aortic root rupture, and aortic root diameter $45 \mathrm{~mm}$ or greater. $^{15}$ 


\section{A systematic approach to improve the outcomes of type A aortic dissection}

Hidefumi Nishida 1, Minoru Tabata 1,2, Toshihiro Fukui 1,3, Yasunori Sato 4, Hajime Kin 1 and Shuichiro Takanashi 1

1. Department of cardiovascular surgery, Sakakibara Heart Insutitute

. Department of cardiovascular surgery, Tokyo Bay Urayasu-Ichikawa Medical center

Department of global clinical research, Graduate school of Medicine, Chiba University

VIDEO 1. Case presentation of ascending aortic replacement for ATAAD. Video available at: http://www.jtcvsonline.org/article/S00225223(17)30560-3/addons.

\section{Statistical Analyses}

All data analyses were performed with JMP 11.0 software (SAS Institute Inc, Cary, NC). Data were expressed as means \pm standard deviations or median and ranges for continuous variables and as numbers (percentages) for categoric variables. Comparisons of continuous variables were tested with unpaired $t$ test or Wilcoxon test, and comparisons of categoric variables were tested with the chi-square test or Fisher exact test. Long-term survival and freedom from aortic reintervention were analyzed using the Kaplan-Meier method and log-rank test. The Cochran-Armitage trend test was used for analysis of trend of proportional variables, such as the rate of operative mortality and that of high-risk patients. The Jonckheere-Terpstra trend test was used for analysis of trend of time from hospital arrival to CPB establishment.

The association between aortic crossclamping and postoperative stroke was examined by multivariable logistic regression analysis. We included covariates that were rationally considered to be related with postoperative stroke: age, sex, previous cardiac surgery, preoperative shock, total arch replacement, CPB time, and FA cannulation. The association between aortic crossclamping and operative mortality was examined by multivariable logistic regression analysis. We included covariates that were rationally considered to be related with operative mortality: age, sex, previous cardiac surgery, malperfusion, preoperative shock, total arch replacement, and CPB time. The association of aortic crossclamping and late aortic reintervention was examined by Cox proportional hazards analysis. We included covariates that were rationally considered to be related with aortic reinterventions: age, total arch replacement, aortic root replacement, resection of primary entry, and DeBakey type II.

\section{RESULTS}

\section{Preoperative Background}

Preoperative data are presented in Table 1. Mean age was $65.5 \pm 13.0$ years, and 334 patients $(47.6 \%)$ were women. A total of 171 patients $(24.4 \%)$ presented to the Sakakibara Heart Institute without a diagnosis and required CT scans after arrival. A total of 617 patients $(87.9 \%)$ underwent surgery within 24 hours after symptom onset. The mean door-to-CPB time was $137.1 \pm 91.0$ minutes, and median time was 115 minutes (range, 33-939 minutes). This time has significantly decreased over a decade (trend test $P<.001)$. Eighteen patients $(2.6 \%)$ who were hemodynamically stable at admission developed cardiac tamponade and shock before surgery.

The ratio of high-risk patients was significantly higher in the nonclamping group $(61.6 \%, 53 / 86)$ than in the crossclamping group $(45.8 \%, 282 / 616)(P<.001)$. The yearly number of surgeries and the ratio of high-risk patients are shown in Figure 1. The number of surgeries has increased over a decade, and the ratio of high-risk patients has significantly increased (trend test $P<.001$ ).

\section{Operative Data}

Operative data are shown in Table 2. Total arch replacement was performed in 242 patients $(34.5 \%)$, and simultaneous aortic root surgery was performed in 82 patients $(11.7 \%)$.

TABLE 1. Preoperative characteristics

\begin{tabular}{|c|c|c|c|c|}
\hline & All $(n=702)$ & Crossclamping $(\mathrm{n}=616)$ & Nonclamping $(\mathbf{n}=86)$ & $P$ value \\
\hline Age, y & $65.5 \pm 13.0$ & $65.3 \pm 0.5$ & $67.5 \pm 1.4$ & .147 \\
\hline Female & $334(47.6 \%)$ & $303(49.2 \%)$ & $31(36.1 \%)$ & .0223 \\
\hline BSA & $1.68 \pm 0.7$ & $1.69 \pm 0.03$ & $1.66 \pm 0.1$ & .800 \\
\hline Hypertension & $593(84.5 \%)$ & $521(84.6 \%)$ & $72(83.7 \%)$ & .837 \\
\hline Diabetes mellitus & $37(5.3 \%)$ & $32(5.2 \%)$ & $5(5.9 \%)$ & .804 \\
\hline Dialysis & $13(1.8 \%)$ & $12(1.9 \%)$ & $1(1.2 \%)$ & .854 \\
\hline Undiagnosed at arrival & $171(24.4 \%)$ & $145(23.5 \%)$ & $26(30.2 \%)$ & .175 \\
\hline Preoperative shock & $115(16.4 \%)$ & $94(15.3 \%)$ & $21(24.4 \%)$ & .0316 \\
\hline Marfan syndrome & $12(1.7 \%)$ & $12(2.0 \%)$ & $0(0 \%)$ & .192 \\
\hline Cerebral malperfusion & $61(8.7 \%)$ & $50(8.1 \%)$ & $11(12.8 \%)$ & .150 \\
\hline Coronary malperfusion & $46(6.6 \%)$ & $38(6.2 \%)$ & $8(9.3 \%)$ & .271 \\
\hline Lower-limb malperfusion & $66(9.4 \%)$ & $51(8.3 \%)$ & $15(17.4 \%)$ & .006 \\
\hline Mesenteric malperfusion & $22(3.1 \%)$ & $20(3.3 \%)$ & $2(2.3 \%)$ & .646 \\
\hline DeBakey type I & $493(70.2 \%)$ & $430(69.7 \%)$ & $63(74.1 \%)$ & .512 \\
\hline DeBakey type II & $93(13.2 \%)$ & $81(13.1 \%)$ & $12(14.1 \%)$ & .836 \\
\hline Previous cardiac surgery & $16(2.3 \%)$ & $9(1.5 \%)$ & $7(8.1 \%)$ & .001 \\
\hline Moderate or severe aortic valve insufficiency & $77(11.6 \%)$ & $70(11.6 \%)$ & $7(8.1 \%)$ & .344 \\
\hline
\end{tabular}

The values of age and BSA are presented as mean \pm standard deviation, and the others are $\mathrm{n}(\%)$. There are some missing data: 13 in BSA, 6 in diabetes mellitus, 6 in mesenteric malperfusion, and 7 in aortic regurgitation. BSA, Body surface area. 


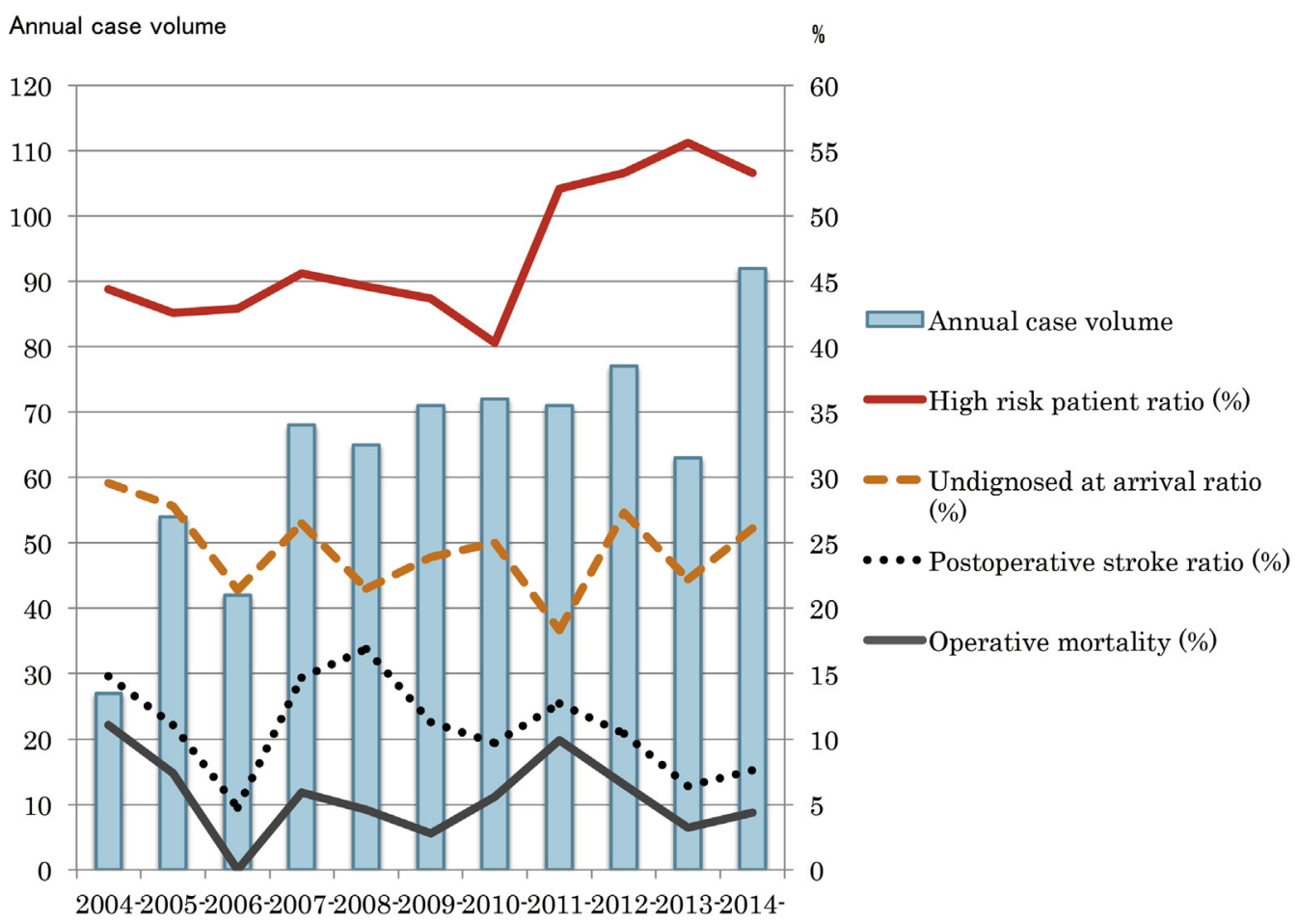

FIGURE 1. Yearly numbers of surgical cases, ratio of high-risk patients, ratio of cases undiagnosed at arrival, postoperative stroke ratio, and operative mortality. Annual case volume has been increasing in this decade. Despite an increasing ratio of high-risk patients, the operative mortality has not changed.

By comparing the operation and CPB times exclusively in isolated ascending or hemiarch replacement cases, the crossclamping group demonstrated shorter operation and CPB times than the nonclamping group. $(236.5 \pm 3.0$ minutes vs $290.6 \pm 8.3$ minutes, $P<.001$ and $131.0 \pm 2.0$ minutes vs $159.7 \pm 5.5$ minutes, $P<.001$ ).

TABLE 2. Operative data

\begin{tabular}{|c|c|c|c|c|}
\hline Variables & All $(\mathbf{n}=702)$ & Crossclamping $(\mathrm{n}=616)$ & Nonclamping $(\mathbf{n}=86)$ & $P$ value \\
\hline \multicolumn{5}{|l|}{ Operative procedures } \\
\hline Ascending or hemiarch replacement & $460(65.5 \%)$ & $414(67.2 \%)$ & $46(53.5 \%)$ & .012 \\
\hline Total arch replacement & $242(34.5 \%)$ & $202(32.8 \%)$ & $40(46.5 \%)$ & \\
\hline \multicolumn{5}{|l|}{ Concomitant procedures } \\
\hline Aortic root replacement & $82(11.7 \%)$ & $75(12.2 \%)$ & $7(8.1 \%)$ & .275 \\
\hline Valve-sparing procedure & $25(3.6 \%)$ & $22(3.6 \%)$ & $3(3.5 \%)$ & .969 \\
\hline CABG & $53(7.6 \%)$ & $43(7.0 \%)$ & $10(11.6 \%)$ & .127 \\
\hline Aortic valve replacement & $16(2.3 \%)$ & $13(2.1 \%)$ & $3(3.5 \%)$ & .423 \\
\hline Resection of primary entry & $554(78.9 \%)$ & $488(79.2 \%)$ & $66(76.7 \%)$ & .600 \\
\hline Operation time & $258.6 \pm 82.5$ & $252.4 \pm 3.3$ & $303.1 \pm 8.7$ & $<.001$ \\
\hline CPB time & $148.0 \pm 63.9$ & $145.0 \pm 2.6$ & $169.8 \pm 6.8$ & $<.001$ \\
\hline \multicolumn{5}{|l|}{ Arterial cannulation site used for perfusion } \\
\hline FA & $615(87.6 \%)$ & $590(95.8 \%)$ & $25(29.1 \%)$ & $<.001$ \\
\hline Apex of left ventricle & $60(8.5 \%)$ & $0(0 \%)$ & $60(69.8 \%)$ & $<.001$ \\
\hline Axillary artery & $16(2.3 \%)$ & $15(2.4 \%)$ & $1(1.7 \%)$ & .460 \\
\hline Ascending aorta & $12(1.7 \%)$ & $11(1.8 \%)$ & $1(1.2 \%)$ & .676 \\
\hline Cannulation site conversion & $24(3.4 \%)$ & $9(1.5 \%)$ & $15(17.4 \%)$ & $<.001$ \\
\hline From FA to apex & $15(2.1 \%)$ & $0(0 \%)$ & $15(18.3 \%)$ & \\
\hline From apex to FA & $5(0.7 \%)$ & $5(0.8 \%)$ & $0(0 \%)$ & \\
\hline From FA to contralateral FA & $3(0.4 \%)$ & $3(0.5 \%)$ & $0(0 \%)$ & \\
\hline From FA to axillary & $1(0.1 \%)$ & $1(0.2 \%)$ & $0(0 \%)$ & \\
\hline Aborted crossclamping & $5(0.7 \%)$ & $5(0.7 \%)$ & Not applicable & - \\
\hline
\end{tabular}

The values of operation time and $\mathrm{CPB}$ time are presented as mean \pm standard deviation, and the others are $\mathrm{n}(\%) . C A B G$, Coronary artery bypass grafting; $C P B$, cardiopulmonary bypass; $F A$, femoral artery. 
TABLE 3. Early surgical outcomes

\begin{tabular}{lccc}
\hline \multicolumn{1}{c}{ Variables } & All $(\mathbf{N}=\mathbf{7 0 2})$ & Crossclamping $(\mathbf{N}=\mathbf{6 1 6})$ & Nonclamping $(\mathbf{n}=\mathbf{8 6})$ \\
\hline Operative mortality & $38(5.4 \%)$ & $30(4.9 \%)$ & $8(9.3 \%)$ \\
Stroke & $76(10.8 \%)$ & $62(10.1 \%)$ & $14(16.3 \%)$ \\
Renal failure & $58(8.3 \%)$ & $47(7.6 \%)$ & $11(12.8 \%)$ \\
Reexploration for bleeding & $41(5.8 \%)$ & $36(5.8 \%)$ & $5(5.8 \%)$ \\
Mediastinitis & $21(3.0 \%)$ & $18(2.9 \%)$ & $3(3.5 \%)$ \\
\hline
\end{tabular}

Values are presented as $\mathrm{n}(\%)$

The details of arterial cannulation site are shown in Table 2. Twenty-four patients required the switch of arterial cannulation site because of malperfusion ( 22 cases), severe aortic regurgitation (1 case), and root rupture (1 case). Aortic crossclamping was placed in 616 patients $(87.7 \%)$. In 5 of those patients, malperfusion occurred after the aortic crossclamping. In those cases, malperfusion was resolved after prompt unclamping $(n=3)$ or changing the clamp site to the proximal ascending aorta $(n=2)$.

\section{Early Outcomes}

Early surgical outcomes are shown in Table 3. Operative mortality was $5.4 \%$ (38/702), and the incidence of stroke was $10.8 \%$ (76/702). There were no significant differences in the incidences of complications and operative mortality between 2 groups. Logistic regression analysis did not reveal that aortic crossclamping is associated with operative mortality (adjusted odds ratio, $0.77 ; 95 \%$ confidence interval $[\mathrm{CI}], 0.32-2.05 ; P=.58)$ and postoperative stroke (adjusted odds ratio, 0.75; 95\% CI, 0.3-1.92; $P=.542$ ). Preoperative shock was the only independent predictor of postoperative stroke (Table 4).

The incidence of stroke in patients with preoperative cerebral malperfusion $(n=61)$ was $29.5 \%$ (18/61), which was significantly higher than in patients without preoperative cerebral malperfusion $(9.0 \%, 58 / 641)$. In the crossclamping group, the incidences of stroke were $26 \%$ (13/ 50) in patients with preoperative cerebral malperfusion and $8.7 \%(49 / 566)$ in patients without preoperative cerebral malperfusion. In the nonclamping group, the incidences of stroke were $45 \%(5 / 11)$ in patients with preoperative

TABLE 4. Impact of aortic crossclamping and other predictors on postoperative stroke

\begin{tabular}{lccr}
\hline \multicolumn{1}{c}{ Risk factors } & Adjusted OR & $\mathbf{9 5} \%$ CI & $\boldsymbol{P}$ value \\
\hline Aortic crossclamping & 0.75 & $0.30-1.92$ & .542 \\
Age & 1.02 & $0.99-1.04$ & .081 \\
Female & 1.12 & $0.65-1.92$ & .700 \\
Preoperative shock & 3.15 & $1.78-5.50$ & $<.001$ \\
Previous cardiac surgery & 2.54 & $0.62-8.57$ & .157 \\
Total arch replacement & 0.89 & $0.50-1.59$ & .707 \\
CPB time & 0.99 & $0.99-1.00$ & .417 \\
FA cannulation & 0.89 & $0.50-1.59$ & .683 \\
\hline
\end{tabular}

$O R$, Odds ratio; $C I$, confidence interval; $C P B$, cardiopulmonary bypass; $F A$, femoral artery. cerebral malperfusion and $12.0 \%(9 / 75)$ in patients without preoperative cerebral malperfusion.

\section{Long-Term Survivals}

The rate of complete follow-up was $94.0 \%$. Overall actuarial survivals were $85.0 \%$ at 5 years and $80.4 \%$ at 7 years in all patients (Figure 2, $A$ ), $86.0 \%$ at 5 years and $82.4 \%$ at 7 years in the crossclamping group, and $78.4 \%$ at 5 years and $69.1 \%$ at 7 years in the nonclamping group $(P<.001)$ (Figure 2, $A)$.

\section{Late Aortic Reinterventions}

Fifty-five patients underwent a total of 58 aortic reinterventions after the primary surgery during the follow-up period. The details of reintervention procedures are shown in Table E1. Freedom rates from aortic reinterventions were $89.8 \%$ at 5 years and $87.5 \%$ at 7 years in all patients (Figure 2, B), 90.3\% at 5 years and $87.5 \%$ at 7 years in the crossclamping group, and $87.1 \%$ at 5 years and $87.1 \%$ at 7 years in the nonclamping group $(P=.758)$ (Figure 2 , $B)$. Cox proportional hazards analysis did not reveal that aortic crossclamping is associated with aortic reinterventions (adjusted hazard ratio, 0.90; 95\% CI, 0.44-2.09; $P=$.799) (Table 5).

\section{DISCUSSION}

\section{Outcomes and Fast Preoperative Management}

To the best of our knowledge, this study is the largest surgical series of ATAAD in a single institute. Our series has demonstrated a low operative mortality of $5.4 \%$, which is better than most of recently reported operative mortalities from large sample studies $(12.7 \%$ to $29.8 \%) .^{1-5}$ In addition, the trend test did not reveal a significant decrease or increase in the operative mortality over the last decade, although the ratio of high-risk patients has been significantly increasing. This fact indicates that our quality of care has been improving over time.

Our long-term survival after emergency surgery for ATAAD is comparable or better than that reported by others, ${ }^{1,5,16-18}$ and our long-term freedom from aortic reinterventions also is comparable or better than in other reports. ${ }^{18-20}$

It is critically important to begin surgery before patients become unstable. ${ }^{21,22}$ Trimarchi and colleagues ${ }^{23}$ in the International Registry of Acute Aortic Dissection reported 

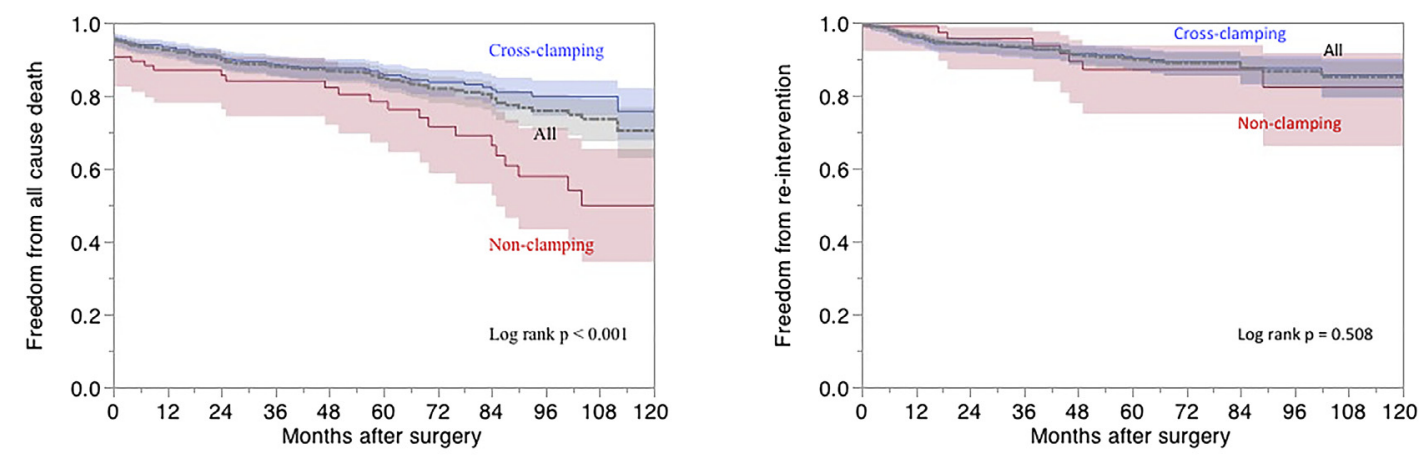

A No.at risk 6

$\begin{array}{lllll}421 & 282 & 160 & 66 & 14 \\ 59 & 46 & 31 & 18 & 4\end{array}$

B No.at risk ${ }_{86}^{616}$

${ }_{56}^{396}$

25

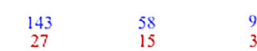

FIGURE 2. A, Freedom from all-cause death. Kaplan-Meier estimates have shown excellent long-term survival after emergency surgery for ATAAD. Log-rank test has shown higher long-term survival in the crossclamping group than in the nonclamping group. B, Freedom from aortic reinterventions. Kaplan-Meier estimates have shown excellent freedom rate from aortic reinterventions after emergency surgery for ATAAD. Log-rank test showed no significant difference in the freedom rate from aortic reinterventions between the crossclamping and nonclamping groups.

that mortality in unstable patients was significantly higher compared with stable patients $(31.4 \%$ vs $16.7 \%)$. ${ }^{23}$ Bavaria and colleagues $^{24}$ reported that surgical outcomes of ATAAD improved with their integrated surgical approach. They transferred the patients directly to the OR from the helipad or emergency department to decrease the interval between diagnosis and surgical intervention. Andersen and colleagues ${ }^{25}$ demonstrated a dramatic improvement in surgical outcomes of ATAAD after implementation of a multidisciplinary thoracic aortic surgery program. Not only surgery per se but also a systematic and sophisticated perioperative approach is key to reduce operative mortality and morbidities. ${ }^{24-27}$

Quick transportation to the OR is critical; however, being in the OR per se or skin incision does not resolve threats of ATAAD, and it is not always easy to establish CPB quickly in ATAAD. Therefore, we chose a door-to-CPB time as a time-related quality index. Our median door-to-CPB time was 115 minutes. This type of time-related quality index has been reported infrequently, and it is difficult to compare those. Harris and colleagues ${ }^{28}$ reported that their median time between diagnosis and surgery was 4.3 hours.

In our analysis apart from this study, we did not reveal the significant association of door-to-CPB time with operative mortality. We think that the positive association was absent

TABLE 5. Impact of aortic crossclamping and other predictors on aortic reinterventions

\begin{tabular}{lccc}
\hline \multicolumn{1}{c}{ Risk factors } & Adjusted HR & $\mathbf{9 5} \% \mathbf{C I}$ & $\boldsymbol{P}$ value \\
\hline Aortic crossclamping & 0.90 & $0.44-2.09$ & .799 \\
Age & 0.97 & $0.95-0.99$ & .006 \\
Total arch replacement & 1.06 & $0.56-1.94$ & .859 \\
Aortic root replacement & 0.54 & $0.18-1.34$ & .197 \\
Resection of primary entry & 0.59 & $0.33-1.07$ & .083 \\
DeBakey type II & 0.76 & $0.23-1.92$ & .591 \\
\hline
\end{tabular}

$H R$, Hazard ratio; $C I$, confidence interval. because door-to-CPB time was influenced by the patient's condition. In our series, 18 patients who were hemodynamically stable at admission developed cardiac tamponade and shock before surgery. This fact indicates the importance of fast transfer to the OR and CPB establishment.

We have kept making small improvements. Although we used to start preparing the OR after the diagnosis has been confirmed, now we start preparing the OR as soon as we receive a phone call from a transferring hospital or ambulance service. We also began using an all-in-one package of surgical instruments and drapes and preconnected CPB circuits so that we have become able to prepare the OR very fast. Once a patient with ATAAD is transferred to the OR, all staffs including surgeons, anesthesiologists, nurses, and perfusionists collaborate effectively to establish $\mathrm{CPB}$ as fast as possible. The trend test showed a significant decrease in our door-to-CPB time over the last decade, which was likely due to our step-by-step quality improvement.

\section{Systematic Cannulation Protocol for Quick Cardiopulmonary Bypass Establishment}

Systematic arterial cannulation site selection contributes to fast establishment of CPB in the OR. The advantages of FA cannulation are technical easiness and speed. In our series, 615 of 634 FA cannulations $(97.0 \%)$ were successful without malperfusion. The left ventricular apex was selected as the second cannulation site in most failure cases. The left ventricular apex cannulation under TEE guidance is a simple and reliable method for proper true lumen perfusion. ${ }^{29}$ This systematic cannulation protocol helps surgeons to make quick decisions and perfusion.

On the other hand, major disadvantages of FA cannulation include embolic stroke and malperfusion due to the retrograde perfusion. Some studies have shown significantly lower incidences of stroke with axillary 
arterial cannulation than FA cannulation. ${ }^{30,31}$ Other studies have shown no significant difference in the incidences of stroke $^{32-34}$ and malperfusion ${ }^{31-34}$ between 2 cannulation sites. The optimal site of cannulation in ATAAD is still controversial.

The incidence of postoperative stroke and malperfusion in our FA cannulation series was $10.8 \%$ and $3.0 \%$, respectively, which are comparable to or better than those of axillary cannulation previously reported. Lee and colleagues $^{34}$ have reported even lower incidences of postoperative stroke $(5.7 \%)$ and malperfusion $(0 \%)$ after FA cannulation. Of note, they excluded patients with severe atherosclerotic disease in the aorta, iliac, and femoral arteries from FA cannulation candidates. They also routinely monitored blood pressure of extremities and evaluated the area ratio of true and false lumens in the descending aorta with TEE to detect malperfusion when initiating CPB. Their and our findings indicate that FA cannulation is safe and effective in ATAAD if it is performed under careful patient selection and intraoperative monitoring of malperfusion.

\section{Impact of Temporary Aortic Crossclamping During Cooling}

Placing temporary aortic crossclamping during cooling is another controversy. In this study, we have shown that temporary aortic crossclamping decreased the operation time without increasing the operative mortality, incidence of stroke, late deaths, and aortic reinterventions. There have been only a few studies focusing on the impact of aortic crossclamping in ATAAD.

David and colleagues ${ }^{7}$ compared the early and late outcomes between 55 patients in whom the aorta was clamped during distal anastomosis and 54 patients in whom the aorta was not clamped and the distal anastomosis was performed under circulatory arrest. This unadjusted comparative study showed that aortic crossclamping group had a higher incidence of stroke and higher rate of patent false lumen on follow-up images. ${ }^{7}$ Lawton and colleagues ${ }^{9}$ recently published similar findings. Immer and colleagues ${ }^{35}$ showed that temporary aortic crossclamping during cooling did not affect the in-hospital mortality or neurologic deficits.

Potential risks of aortic crossclamping include traumatic injuries of diseased aorta and thrombotic events due to mobilization of clot in the false lumen, creating a new intimal tear or malperfusion due to pressurization of the false lumen. It is important to monitor arterial pressure, cerebral oxygen saturation, and TEE findings of the aorta for confirming proper perfusion after crossclamping. We have experienced and appropriately managed 5 cases $(0.8 \%)$ of malperfusion occurring after crossclamping the aorta. Our multivariable analyses showed no association between aortic crossclamping and incidences of stroke or aortic reinterventions. Temporary aortic crossclamping during cooling seems efficient and safe if it is performed with careful maneuvers and monitoring as described.

\section{Stroke}

The incidence of stroke after ATAAD surgery has been reported as $2 \%$ to $16 \%$ in recent studies. ${ }^{3,4,32,36}$ Some may think that our stroke rate $(10.8 \%)$ is relatively high. As our multivariable analysis showed, we think that preoperative shock status is largely related to the incidence of stroke. The rate of preoperative shock was $16 \%$ in our series. One series demonstrating the low incidence of stroke $(2 \%)$ included only a small number of preoperative shock $(3 \%){ }^{36}$ On the other hand, another series included a large number of preoperative shock patients $(22 \%)$ and demonstrated the high incidence of stroke $(13 \%){ }^{4}$

The incidence of stroke in patients with preoperative cerebral malperfusion was high $(29.5 \%$ in all patients, $26 \%$ in the crossclamping group, $45.5 \%$ in the nonclamping group). The safety of aortic crossclamping in those patients is another concern. Although it is difficult to assess this clinical question adjusting all bias with this small sample size, our results do not indicate that aortic crossclamping increase the risk of new stroke in patients with preoperative cerebral malperfusion.

\section{Study Limitations}

Our present study has some limitations due to its retrospective and single-center study nature. There are significant differences in the background and perfusion strategy between the clamping and nonclamping groups. Although we tried to minimize the bias by using the multivariable analysis, there is likely to be unadjusted bias. As mentioned earlier, there were some reasons why we did not clamp the aorta in each case. Those indicators against aortic crossclamping are potential confounding factors, and there would be no sample if we tried to adjust all of those indicators. A prospective randomized study would be ideal to determine the optimal surgical strategy for ATAAD.

There is potential patient selection bias. We essentially operate on all patients with ATAAD unless a patient rejected surgery or is comatose without pupillary reflex. ${ }^{14}$ Most of our patients undergoing dissection were referred from other hospitals, and patients who are deferred from surgery by a referring physician or those who reject undergoing surgery are not transferred to us.

\section{CONCLUSIONS}

Emergency surgery for ATAAD based on our systematic approach demonstrated excellent early and late outcomes. The operative mortality has been stably low over the last decade despite the increase in high-risk patients. Under the principle of open distal anastomosis and primary entry 
resection, temporary aortic crossclamping during cooling decreased the operation time without increasing the operative mortality, incidence of stroke, late deaths, and aortic reinterventions.

\section{Conflict of Interest Statement}

Authors have nothing to disclose with regard to commercial support.

\section{References}

1. Stevens LM, Madsen JC, Isselbacher EM, Khairy P, MacGillivray TE, Hilgenberg AD, et al. Surgical management and long-term outcomes for acute ascending aortic dissection. J Thorac Cardiovasc Surg. 2009;138:1349-57.

2. Chikwe J, Cavallaro P, Itagaki S, Seigerman M, DiLuozzo G, Adams DH. National outcomes in acute aortic dissection: influence of surgeon and institutional volume on operative mortality. Ann Thorac Surg. 2013;95:1563-9.

3. Omura A, Miyahara S, Yamanaka K, Sakamoto T, Matsumori M, Okada K, et al. Early and late outcomes of repaired acute DeBakey type I aortic dissection after graft replacement. J Thorac Cardiovasc Surg. 2016;151:341-8.

4. Easo J, Weigang E, Hölzl PP, Horst M, Hoffmann I, Blettner M, et al. Influence of operative strategy for the aortic arch in DeBakey type I aortic dissection: analysis of the German Registry for Acute Aortic Dissection Type A. J Thorac Cardiovasc Surg. 2012;144:617-23.

5. Tsai TT, Fattori R, Trimarchi S, Isselbacher E, Myrmel T, Evangelista A, et al. Long-term survival in patients presenting with type A acute aortic dissection Insights from the International Registry of Acute Aortic Dissection (IRAD). Circulation. 2006;114(1 Suppl):I-350-6.

6. Bonser RS, Ranasinghe AM, Loubani M, Evans JD, Thalji NM, Bachet JE, et al. Evidence, lack of evidence, controversy, and debate in the provision and performance of the surgery of acute type A aortic dissection. J Am Coll Cardiol. 2011; 58:2455-74.

7. David TE, Armstrong S, Ivanov J, Barnard S. Surgery for acute type A aortic dissection. Ann Thorac Surg. 1999;67:1999-2001; discussion 2014-9.

8. Erbel R, Aboyans V, Boileau C, Bossone E, Bartolomeo RD, Eggebrecht H, et al. 2014 ESC Guidelines on the diagnosis and treatment of aortic diseases: document covering acute and chronic aortic diseases of the thoracic and abdominal aorta of the adult. The Task Force for the Diagnosis and Treatment of Aortic Diseases of the European Society of Cardiology (ESC). Eur Heart J. 2014;35: 2873-926.

9. Lawton JS, Liu J, Kulshrestha K, Moon MR, Damiano RJ Jr, Maniar H, et al. The impact of surgical strategy on survival after repair of type A aortic dissection. J Thorac Cardiovasc Surg. 2015;150:294-301.

10. Mehta RH, Suzuki T, Hagan PG, Bossone E, Gilon D, Llovet A, et al. Predicting death in patients with acute type A aortic dissection. Circulation. 2002;105: 200-6.

11. Girdauskas E, Kuntze T, Borger MA, Falk V, Mohr FW. Surgical risk of preoperative malperfusion in acute type A aortic dissection. J Thorac Cardiovasc Surg. 2009; 138:1363-9.

12. Shimokawa T, Takanashi S, Ozawa N, Itoh T. Management of intraoperative malperfusion syndrome using femoral artery cannulation for repair of acute type A aortic dissection. Ann Thorac Surg. 2008;85:1619-24.

13. Matsuyama S, Tabata M, Shimokawa T, Matsushita A, Fukui T, Takanashi S. Outcomes of total arch replacement with stepwise distal anastomosis technique and modified perfusion strategy. J Thorac Cardiovasc Surg. 2012;143:1377-81.

14. Matsushita A, Tabata M, Fukui T, Sato Y, Matsuyama S, Shimokawa T, et al. Outcomes of contemporary emergency open surgery for type A acute aortic dissection in elderly patients. J Thorac Cardiovasc Surg. 2014;147:290-4.

15. Nishida H, Tabata M, Fukui T, Takanashi S. Surgical strategy and outcome for aortic root in patients undergoing repair of acute type A aortic dissection. Ann Thorac Surg. 2016;101:1464-9.

16. Ehrlich MP, Ergin MA, McCullough JN, Lansman SL, Galla JD, Bodian CA, et al. Results of immediate surgical treatment of all acute type A dissections. Circulation. 2000;102(Suppl 3):III248-52.

17. Chiappini B, Schepens M, Tan E, Dell'Amore A, Morshuis W, Dossche K, et al. Early and late outcomes of acute type A aortic dissection: analysis of risk factors in 487 consecutive patients. Eur Heart J. 2005;26:180-6.
18. Rylski B, Milewski RK, Bavaria JE, Vallabhajosyula P, Moser W, Szeto WY, et al. Long-term results of aggressive hemiarch replacement in 534 patients with type A aortic dissection. J Thorac Cardiovasc Surg. 2014;148: 2981-5.

19. Concistrè G, Casali G, Santaniello E, Montalto A, Fiorani B, Dell'Aquila A, et al. Reoperation after surgical correction of acute type A aortic dissection: risk factor analysis. Ann Thorac Surg. 2012;93:450-5.

20. Zierer A, Voeller RK, Hill KE, Kouchoukos NT, Damiano RJ, Moon MR. Aortic enlargement and late reoperation after repair of acute type A aortic dissection. Ann Thorac Surg. 2007:84:479-87.

21. Hagan PG, Nienaber CA, Isselbacher EM, Bruckman D, Karavite DJ, Russman PL, et al. The International Registry of Acute Aortic Dissection (IRAD): new insights into an old disease. JAMA. 2000;283:897-903.

22. Nienaber CA, Eagle KA. Aortic dissection: new frontiers in diagnosis and management part I: from etiology to diagnostic strategies. Circulation. 2003;108 628-35.

23. Trimarchi S, Nienaber CA, Rampoldi V, Myrmel T, Suzuki T, Mehta RH, et al. Contemporary results of surgery in acute type A aortic dissection: the International Registry of Acute Aortic Dissection experience. J Thorac Cardiovasc Surg. 2005;129:112-22.

24. Bavaria JE, Pochettino A, Brinster DR, Gorman RC, McGarvey ML, Gorman JH, et al. New paradigms and improved results for the surgical treatment of acute type A dissection. Ann Surg. 2001;234:336-42.

25. Andersen ND, Ganapathi AM, Hanna JM, Williams JB, Gaca JG, Hughes GC. Outcomes of acute type a dissection repair before and after implementation of a multidisciplinary thoracic aortic surgery program. J Am Coll Cardiol. 2014; 63:1796-803.

26. Harris KM, Strauss CE, Duval S, Unger BT, Kroshus TJ, Inampudi S, et al. Multidisciplinary standardized care for acute aortic dissection design and initial outcomes of a regional care model. Circ Cardiovasc Qual Outcomes. 2010;3: 424-30.

27. Westaby S, Saito S, Katsumata T. Acute type A dissection: conservative methods provide consistently low mortality. Ann Thorac Surg. 2002;73:707-13.

28. Harris KM, Strauss CE, Eagle KA, Hirsch AT, Isselbacher EM, Tsai TT, et al. Correlates of delayed recognition and treatment of acute type a aortic dissection: the International Registry of Acute Aortic Dissection (IRAD). Circulation. 2011; 124:1911-8.

29. Matsushita A, Manabe S, Tabata M, Fukui T, Shimokawa T, Takanashi S. Efficacy and pitfalls of transapical cannulation for the repair of acute type A aortic dissection. Ann Thorac Surg. 2012;93:1905-9.

30. Benedetto U, Mohamed H, Vitulli P, Petrou M. Axillary versus femoral arterial cannulation in type A acute aortic dissection: evidence from a meta-analysis of comparative studies and adjusted risk estimates. Eur J Cardiothorac Surg. 2015;48:953-9.

31. Ren Z, Wang Z, Hu R, Wu H, Deng H, Zhou Z, et al. Which cannulation (axillary cannulation or femoral cannulation) is better for acute type A aortic dissection repair? A meta-analysis of nine clinical studies. Eur J Cardiothorac Surg. 2015;47:408-15.

32. Etz CD, von Aspern K, da Rocha E, Silva J, Girrbach FF, Leontyev S, et al. Impact of perfusion strategy on outcome after repair for acute type A aortic dissection. Ann Thorac Surg. 2014;97:78-85.

33. Reuthebuch O, Schurr U, Hellermann J, Prêtre R, Künzli A, Lachat M, et al. Advantages of subclavian artery perfusion for repair of acute type A dissection. Eur J Cardiothorac Surg. 2004;26:592-8.

34. Lee HK, Kim GJ, Cho JY, Lee JT, Park I, Lee YO. Comparison of the outcomes between axillary and femoral artery cannulation for acute type A aortic dissection. Korean J Thorac Cardiovasc Surg. 2012;45:85-90.

35. Immer FF, Aydin NB, Lütolf M, Krähenbühl ES, Stalder M, Englberger L, et al. Does aortic crossclamping during the cooling phase affect the early clinical outcome of acute type A aortic dissection? J Thorac Cardiovasc Surg. 2008; 136:1536-40.

36. Ma WG, Zhang W, Wang LF, Zheng J, Ziganshin BA, Charilaou P, et al. Type A aortic dissection with arch entry tear: Surgical experience in 104 patients over a 12-year period. J Thorac Cardiovasc Surg. 2016;151:1581-92.

Key Words: acute type A aortic dissection, aortic crossclamping, femoral artery cannulation, open distal anastomosis 
TABLE E1. Details of aortic reinterventions

\begin{tabular}{|c|c|c|c|c|c|}
\hline First procedure & $\mathbf{N}$ & Second & $\mathbf{N}$ & Third & \\
\hline \multirow[t]{6}{*}{ Ascending/hemiarch replacement } & 405 & Total arch replacement & 19 & Descending replacement (open) & \\
\hline & & & & Descending repair (TEVAR) & \\
\hline & & Descending replacement (open) & 4 & & \\
\hline & & Descending repair (TEVAR) & 1 & & \\
\hline & & Thoracoabdominal replacement & 1 & & \\
\hline & & Aortic root replacement & 2 & & \\
\hline \multirow[t]{4}{*}{ Total arch replacement } & 215 & Descending replacement (open) & 13 & Thoracoabdominal replacement & \\
\hline & & Descending repair (TEVAR) & 1 & & \\
\hline & & Aortic root replacement & 3 & & \\
\hline & & Thoracoabdominal replacement & 1 & & \\
\hline \multirow{2}{*}{$\begin{array}{l}\text { Aortic root and ascending/hemiarch } \\
\text { replacement }\end{array}$} & 55 & Total arch replacement & 2 & Thoracoabdominal replacement & \\
\hline & & Descending replacement (open) & 2 & & \\
\hline Aortic root and total arch replacement & 27 & Descending replacement (open) & 1 & & \\
\hline Total & 702 & & 54 & & \\
\hline
\end{tabular}

TEVAR, Thoracic endovascular aortic repair. 